\title{
Pressure Pulsation Signal Analysis for Centrifugal Compressor Blade Crack Determination
}

\author{
Hongkun Li, ${ }^{1}$ Xuefeng Zhang, ${ }^{1}$ Xiaowen Zhang,, Shuhua Yang, ${ }^{2}$ and Fujian Xu ${ }^{1}$ \\ ${ }^{1}$ School of Mechanical Engineering, Dalian University of Technology, No. 2 Linggong Road, Dalian 116024, China \\ ${ }^{2}$ Shenyang Blower Works Group Corporation, Shenyang 110869, China \\ Correspondence should be addressed to Hongkun Li; lihk@dlut.edu.cn
}

Received 28 March 2014; Revised 31 May 2014; Accepted 16 June 2014; Published 19 August 2014

Academic Editor: Ruqiang Yan

Copyright (c) 2014 Hongkun Li et al. This is an open access article distributed under the Creative Commons Attribution License, which permits unrestricted use, distribution, and reproduction in any medium, provided the original work is properly cited.

\begin{abstract}
Blade is a key piece of component for centrifugal compressor. But blade crack could usually occur as blade suffers from the effect of centrifugal forces, gas pressure, friction force, and so on. It could lead to blade failure and centrifugal compressor closing down. Therefore, it is important for blade crack early warning. It is difficult to determine blade crack as the information is weak. In this research, a pressure pulsation (PP) sensor installed in vicinity to the crack area is used to determine blade crack according to blade vibration transfer process analysis. As it cannot show the blade crack information clearly, signal analysis and empirical mode decomposition (EMD) are investigated for feature extraction and early warning. Firstly, signal filter is carried on PP signal around blade passing frequency (BPF) based on working process analysis. Then, envelope analysis is carried on to filter the BPF. In the end, EMD is carried on to determine the characteristic frequency (CF) for blade crack. Dynamic strain sensor is installed on the blade to determine the crack CF. Simulation and experimental investigation are carried on to verify the effectiveness of this method. The results show that this method can be helpful for blade crack classification for centrifugal compressors.
\end{abstract}

\section{Introduction}

With the development of the society, centrifugal compressor has been widely used in modern industry such as petroleum, chemicals, metallurgy, and aerospace field as an important fluid machine [1]. Meanwhile, centrifugal compressors are developing to be large in scale, high in speed, and automatic in operation [2]. However, the blade failure usually emerges. As the most important part, the impeller transforms kinetic energy into pressure energy. But the impeller suffers from the effect of centrifugal forces, gas pressure, and friction force which usually lead to cracks. According to statistical analysis, 65\% centrifugal compressor malfunctions are closely related to the blades. In addition, $40 \%$ blade fatigue failures are not fully understood so far [3]. Examples of blade cracks are shown in Figure 1. Fluid-induced vibration is an important factor for blade fatigue failures. It contains acoustic resonance, unsteady flow, rotating stalls, and flutter $[4,5]$. Due to the high-velocity flow through the centrifugal compressor and rotating impeller, high-pressure fluctuations occur in the cavity of compressor which could lead the impeller to irregular vibration. Pressure fluctuation acts on the impeller, leading to stress convergence and cracks in the blades. The growing crack will cause blade failure, which results in catastrophes.

There are many reasons for cracks on the blades of compressor. Blade cracks are mainly associated with the detection of material, production process, working condition, and high cycle fatigue. So far, researches were mainly concentrated on the defects in the material, processing, and manufacture of impeller causing high fatigue failure. Lourenço investigated the failure of blades [6]. Kermanpur et al. analyzed the failure mechanism of compressor blades made of Ti6Al4V. The results showed fretting fatigue mechanism is the main cause of several premature failures of Ti6Al4V alloyed compressor blades [7]. In recent years, blade cracks caused by excessive alternating stress induced by air-excited vibration have drawn more and more attention from the researchers. 

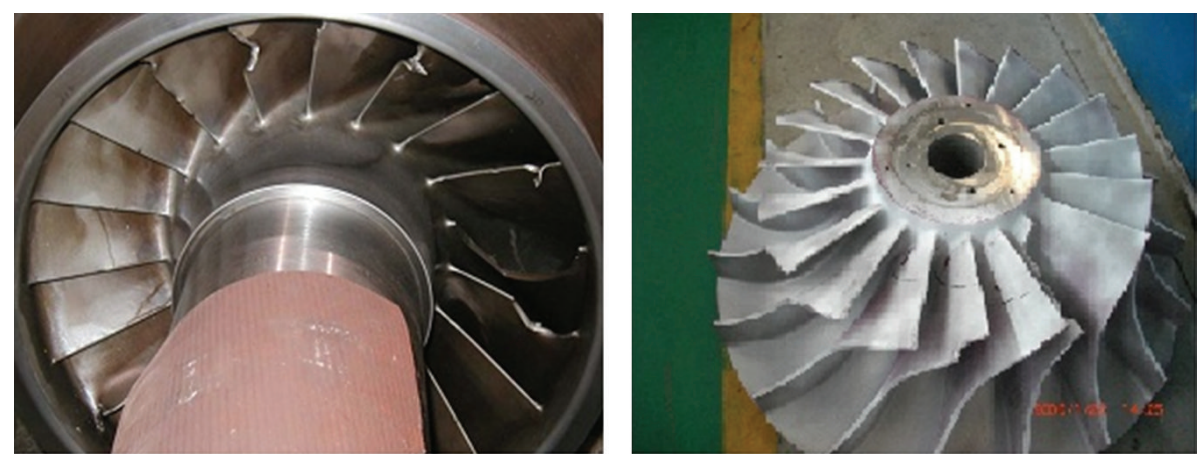

FIgURE 1: Pictures of centrifugal compressor blade cracks.

In 2007, Eisinger studied the acoustic fatigue which is the coincidence of impeller structural and cavity acoustic modes. The results indicated that the acoustic fatigue would significantly increase the amplitude of vibration and damage the blades [8]. Investigation on alternating stress can be helpful to prevent or reduce the damage from blade cracks [9]. Therefore, condition monitoring and pattern classification are important to prevent blades from failure as well as blade crack detection which could ensure safe operation of the compressor.

It is well known that blade cracks will result in breakdown or even serious accidents of the whole set for centrifugal compressor. It can even lead to heavy losses for a factory. Moreover, personal safety must be considered because the tangential velocity of breakdown blade can be up to $450 \mathrm{~m} / \mathrm{s}$. Therefore, incipient classification of blade crack becomes more and more important than ever before. Traditionally, displacement sensors are introduced to monitor shaft vibration. Meanwhile, vibration-based condition monitoring is also used in shaft crack classification $[10,11]$. But it is difficult to recognize shaft cracks only by vibration signals. Moreover, it is impossible to provide any information to characterize blade crack condition from the shaft vibration signals, making blade crack classification more difficult than shaft crack identification. Different methods for blade condition classification have been investigated by many researchers. Liu et al. studied the malfunction identification method of fan blade crack classification by using wavelet packet analysis [12]. Though the structure is similar to centrifugal compressor and fan, centrifugal compressor has good stiffness as the typical difference. Rama Rao and Dutta studied blade crack condition classification for gas turbine blade recognition by using vibration signal information [13]. Yang et al. proposed the auditory spectrum feature extraction using the support vector machine to identify the malfunction of fans [14]. Witek studied the experimental crack propagation for gas turbine blades via vibration signals in laboratory but it was not in a close-loop test-rig [15]. At the same time, some researchers studied wind turbine blade crack classification by using wavelet analysis, scalogram, and so on [16-18]. But it is different from centrifugal compressor blade working condition in speed and load. All these investigations are helpful for blade crack classification, but further study for early warning of centrifugal compressor blade is required. At the same time, air flow experiment is more important for blade condition analysis in real working conditions.

Pressure pulsation (PP) generated by the interference between rotating blades and the stationary vanes contains much information about the blade working conditions and has been used for blade status conditions analysis [19]. However, the crack information in PP signal is weak, so it is difficult to identify patterns just according to time or frequency information, especially for the incipient blade crack condition. Further feature extraction methods are urgently needed for better information collection. Empirical mode decomposition (EMD) is an effective tool for nonstationary signal analysis, which has been widely applied in rolling element bearings and gearbox fault diagnosis. It has great advantage and adaptability in the mechanical fault diagnosis and feature extraction. EMD is a new time-frequency signal analysis method proposed by the scientist of National Aeronautics and Space Administration (NASA) Huang et al. in recent years [20]. This method has been broadly investigated by many researchers since it was provided. It has been applied in different areas for fault diagnosis. Parey et al. used EMD statistical method to detect incipient fault of the gears [21]. Loutridis applied the instantaneous energy density and EMD to monitor and diagnose the gear fault [22]. Liu et al. detected the gear incipient fault with EMD and they found that the result was better than that of wavelet decomposition [23]. EMD can also be used in machine fault diagnosis based on concrete analysis of specific issues. The vibration of blade crack generates a characteristic frequency (CF) which can be modulated into blade passing frequency (BPF). Therefore, EMD can be applied to determine the CF of blade cracks despite of the noise interference in practical working centrifugal compressor. It is also similar to gearbox fault diagnosis problems.

In this paper, PP signals are used for blade working condition classification by using EMD. Experiments are carried on to verify the effectiveness of this method in a test-rig. To verify the effectiveness of this method, strain testing is also carried on for the blade crack analysis. The structure of this paper is as follows. Section 2 introduces the theory of feature extraction for blade crack classification. Section 3 presents the simulation signal analysis. Section 4 
describes our experimental setup for blade crack monitoring. Section 5 demonstrates PP signal analysis for blade condition classification. Section 6 gives concluding remarks.

\section{Theory and Method}

2.1. Empirical Mode Decomposition. EMD is developed based on instantaneous frequency calculation. It has been considered a very useful tool for the analysis of nonstationary and nonlinear signals [20]. For an arbitrary time series $X(t)$, it can decompose the original into many narrow-band components, each component known as intrinsic mode functions. An intrinsic mode function is used to convert it into a practically useful instantaneous frequency. The intrinsic mode function satisfies two conditions: (1) in the whole range of a data set, the number of the extreme must be equal to the number of the zero crossing points or the difference between them must be one; (2) at any given time, the mean value of the local positive extreme is equal to that of the local negative extreme. An arbitrary nonstationary and nonlinear signal can be decomposed into a series of components satisfied with the intrinsic mode function by using the local wave decomposition method. It is a sifting process and can be written as

$$
\begin{gathered}
X(t)-C_{1}(t)=r_{1}(t) \\
r_{1}(t)-C_{2}(t)=r_{2}(t) \\
\vdots \\
r_{n-1}(t)-C_{n}(t)=r_{n}(t) .
\end{gathered}
$$

The original data can be decomposed into an $n$-series of intrinsic mode components plus a residual component $r_{n}$. The residual can be either a variable or a constant. Thus, the original signal can be expressed as

$$
X(t)=\sum_{i=1}^{n} c_{i}(t)+r_{n}(t) .
$$

After EMD, intrinsic mode function (IMF) can be obtained. FFT can be used on different IMFs analysis for CF determination. EMD can be looked as a filter on feature determination. Therefore, it is helpful to obtain the CF.

2.2. Blade Characteristic. In general, centrifugal compressor casing vibration and radiation noise are closely related to blade BPF and its harmonics. It is also generated by the interference between rotor and stator during blade high speed rotation. BPF has high energy in the pressure frequency spectrum. It is the main source of centrifugal compressor noise. Its value can be determined by shaft speed multiplying the number of blade. BPF can be calculated by

$$
\mathrm{BPF}=\frac{\mathrm{RPM}}{60} \times N,
$$

where RPM is the shaft speed and $N$ is the number of blades on the impeller.

BPF is the interference between rotator and stator. As $\mathrm{BPF}$ is a high frequency component, the low frequency

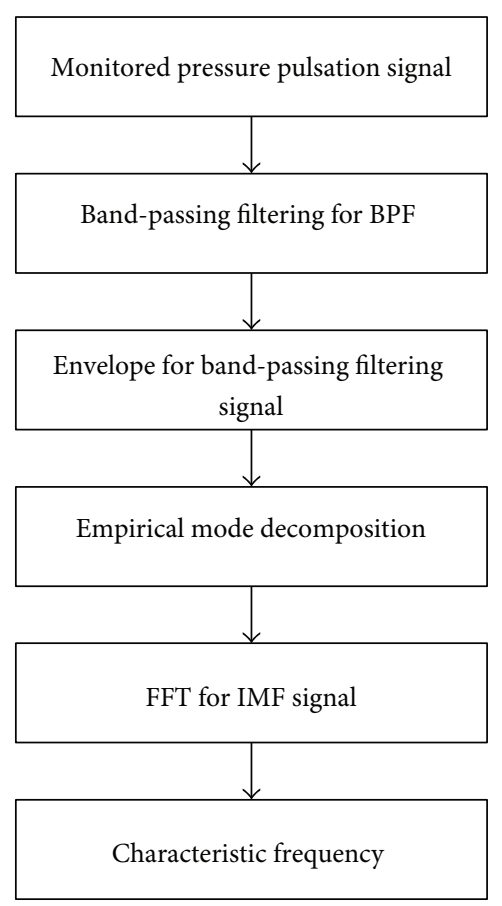

FIGURE 2: Flowchart for blade crack classification using PP signal.

components for blade nonorder vibration can be modulated to $\mathrm{BPF}$ during rotation. The modulation information will appear as the sideband frequency of the BPF. For unbalanced rotor conditions, SF will also be modulated to the BPF giving a sideband frequency around the BPF for unbalanced condition. Sideband frequency could be used to determine the modulated CF. The sideband frequency produced for blade cracks is different from SF. It can be used to warn blade crack. It does not mean that there is a blade with cracks if SF is the sideband frequency for BPF. It is also difficult to classify $\mathrm{CF}$ just according to the spectrum for the incipient crack as the magnitude of the blade vibration is weak compared with the amplitude of BPF. Therefore, effective feature extraction is urgently needed for blade crack analysis.

2.3. Blade Crack Characteristic Frequency Determination. The steps for CF determination are shown in Figure 2. Firstly, PP is monitored based on the best suitable position according to blade crack classification. This is also a key step to determine the crack information because the sensor location has a direct effect on classification accuracy. Secondly, band-pass filter is applied on signal analysis. Envelope analysis is used to filter BPF signal. Then, IMFs can be obtained by using EMD. Fast Fourier transform is used on different IMFs. In the end, CF for blade crack can be obtained. Blade strain is used to verify the effectiveness of this method.

\section{Simulation Signal Analysis}

For a amplitude modulation signal $\operatorname{sig}(t)$, it can be expressed as

$$
\operatorname{sig}(t)=A\left(1+B \cos \left(2 \pi F_{e} t\right)\right) \sin \left(2 \pi F_{c} t\right),
$$




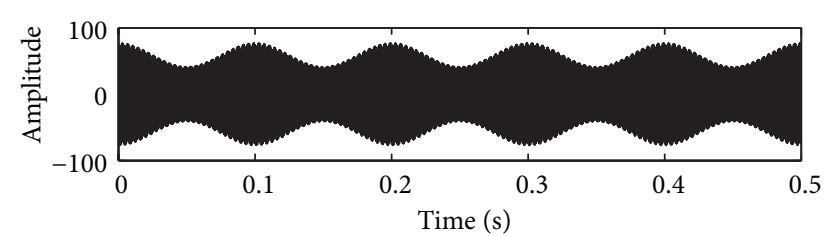

(a)

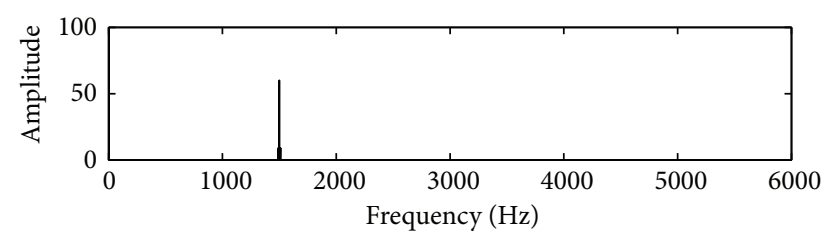

(b)

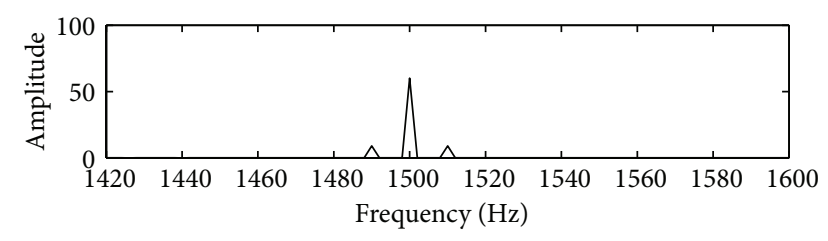

(c)

FIgURE 3: Signal demodulation analysis: (a) time domain signal for the simulation signal; (b) spectrum analysis for the simulation signal; (c) enlarged frequency area for the carrier frequency area.

where, $F_{c}=1500 \mathrm{~Hz}, F_{e}=10 \mathrm{~Hz}, A=60$, and $B=$ 0.3. $F_{c}$ and $F_{e}$ correspond to carrier frequency and modulation frequency, respectively. The corresponding sampling frequency is $10,240 \mathrm{~Hz}$ for the simulation signal. Based on (4), an amplitude modulation signal can be obtained as shown in Figure 3(a). Fourier spectrum analysis is shown in Figure 3(b). The main frequency is $1500 \mathrm{~Hz}$. The modulated frequency $10 \mathrm{~Hz}$ can be obtained by enlarging the frequency domain around the carrier $1500 \mathrm{~Hz}$ frequency shown in Figure 3(c). It is obvious for the sideband frequency around the carrier frequency if there is no noise interference in the signal.

Strong noise interference is added to the simulation signal as the characteristic information is usually overwhelmed by noise under practical working conditions. The obtained signal is shown in Figure 4(a). In the frequency spectrum analysis, there is clear broad frequency band noise effect shown in Figure 4(b). To determine the modulated signal, the enlargement for carrier frequency area in the spectrum is shown in Figure 4(c). Obviously, the enlarged frequency area is not clear due to the noise interference. The noise interference has an effect on the CF determination; therefore, it is difficult to classify the CF just according to sideband frequency spectrum analysis if there is strong noise interference.

Signal filter is used for the monitored signal around BPF. The filter band is $1400-1600 \mathrm{~Hz}$. EMD is applied on the filter signal. IMFs can be obtained as shown in Figure 5. There is not any clear modulated frequency $10 \mathrm{~Hz}$ for every IMFs as shown in Figure 6. Envelope method is applied to the filter signal to filter BPF interference. EMD method is also applied on the envelope signal and IMFs can be obtained as shown

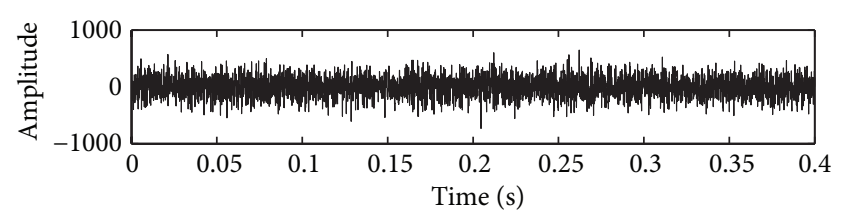

(a)

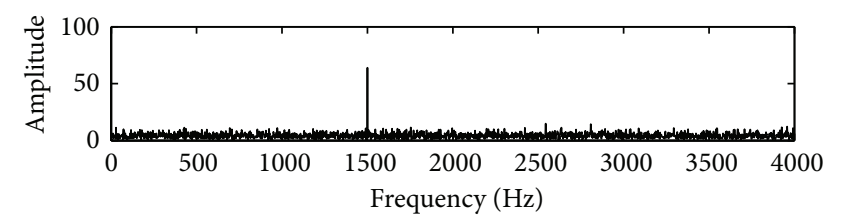

(b)

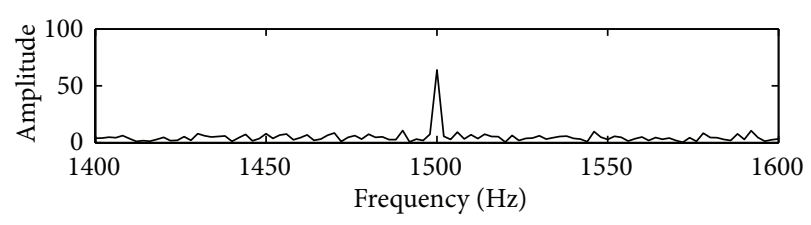

(c)

FIGURE 4: Frequency spectrum analysis: (a) time domain signal for the noise interference signal; (b) spectrum analysis for the noise interference signal; (c) enlarged frequency area for the carrier frequency area.

in Figure 7. But there is clear modulated frequency $10 \mathrm{~Hz}$ as shown in Figure 8, the 5th IMFs based on EMD.

Based on above analysis, it can be convenient to determine the modulated frequency though there is strong noise interference. According to above analysis process, the blade nonorder vibration can be also monitored as it has the same property for simulation signal. Therefore, experimental verification is investigated in this research for blade nonorder vibration classification.

\section{Experimental Test-Rig}

4.1. Testing-Rig. To verify the effectiveness of this method, an experiment was carried on blade crack condition analysis by using the method based on PP signals analysis in a test-rig. The schematic diagram for the test-rig is shown in Figure 9. It contains an electric motor, fluid coupling, gearbox, and impeller. The impeller is a semiclosed one with $800 \mathrm{~mm}$ diameter. It is an experimental impeller for performance testing. By using fluid coupling, the rotating speed for impeller varies from 500 RPM to 9000 RPM. With the speed-up gearbox, the rotation speed of impeller can meet the designed one. The ratio between the driving and driven gears is $126 / 43=2.93$. The experimental picture and hole in the diffuser for installing PP sensor are shown in Figure 10. The crack length during the experiment is $70 \mathrm{~mm}$. PP, vibration, shaft speed sensors are installed to monitor the working process. There are 13 blades in this semiopen impeller. In this experiment, the speed of the impeller is $4500 \mathrm{RPM}$ and $5000 \mathrm{RPM}$. The SF and BPF parameters are shown in Table 1. 



FIGURE 5: Time domain wave of IMFs based on EMD for the filter signal.
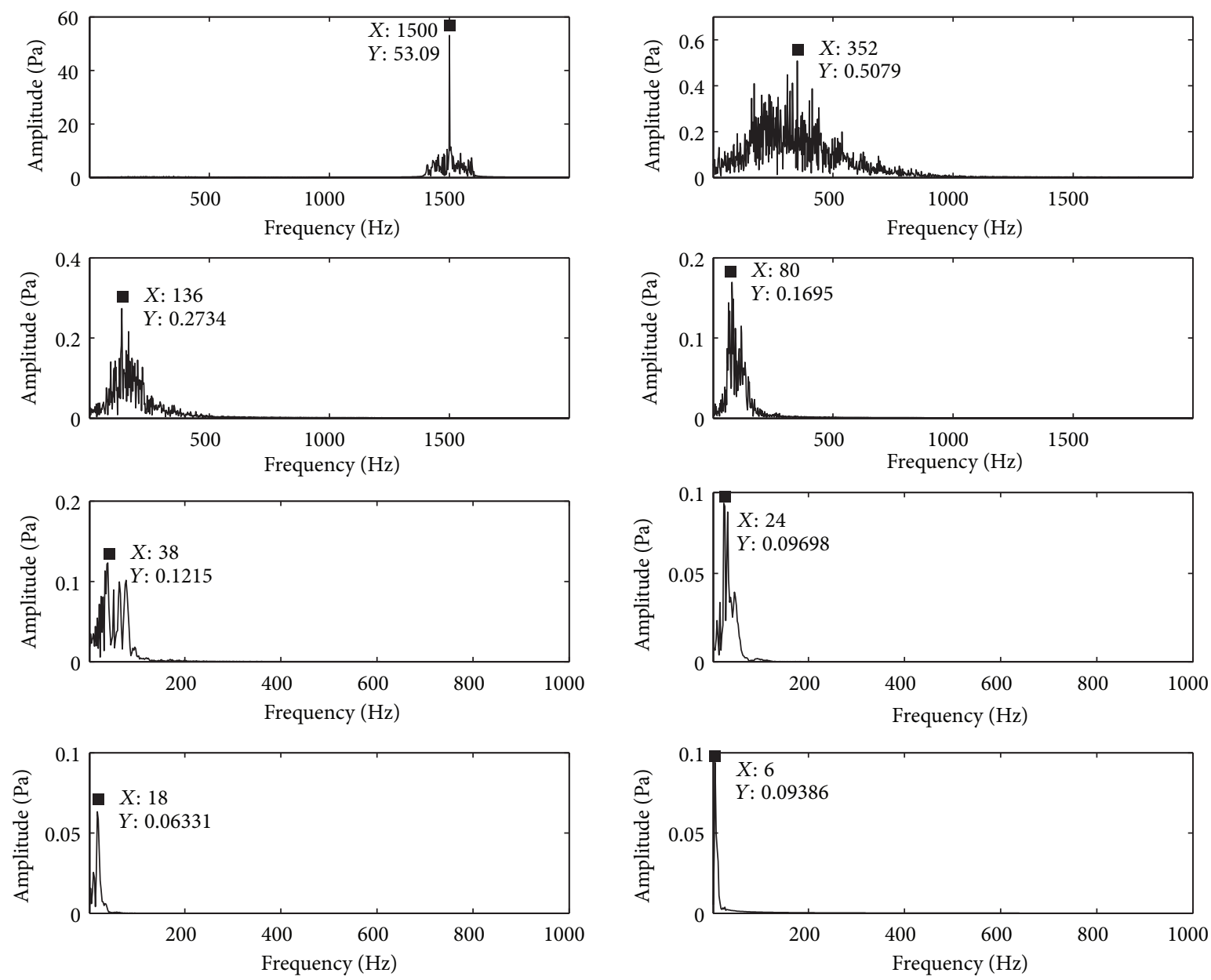

Figure 6: Spectrum of IMFs based on EMD for the filter signal. 

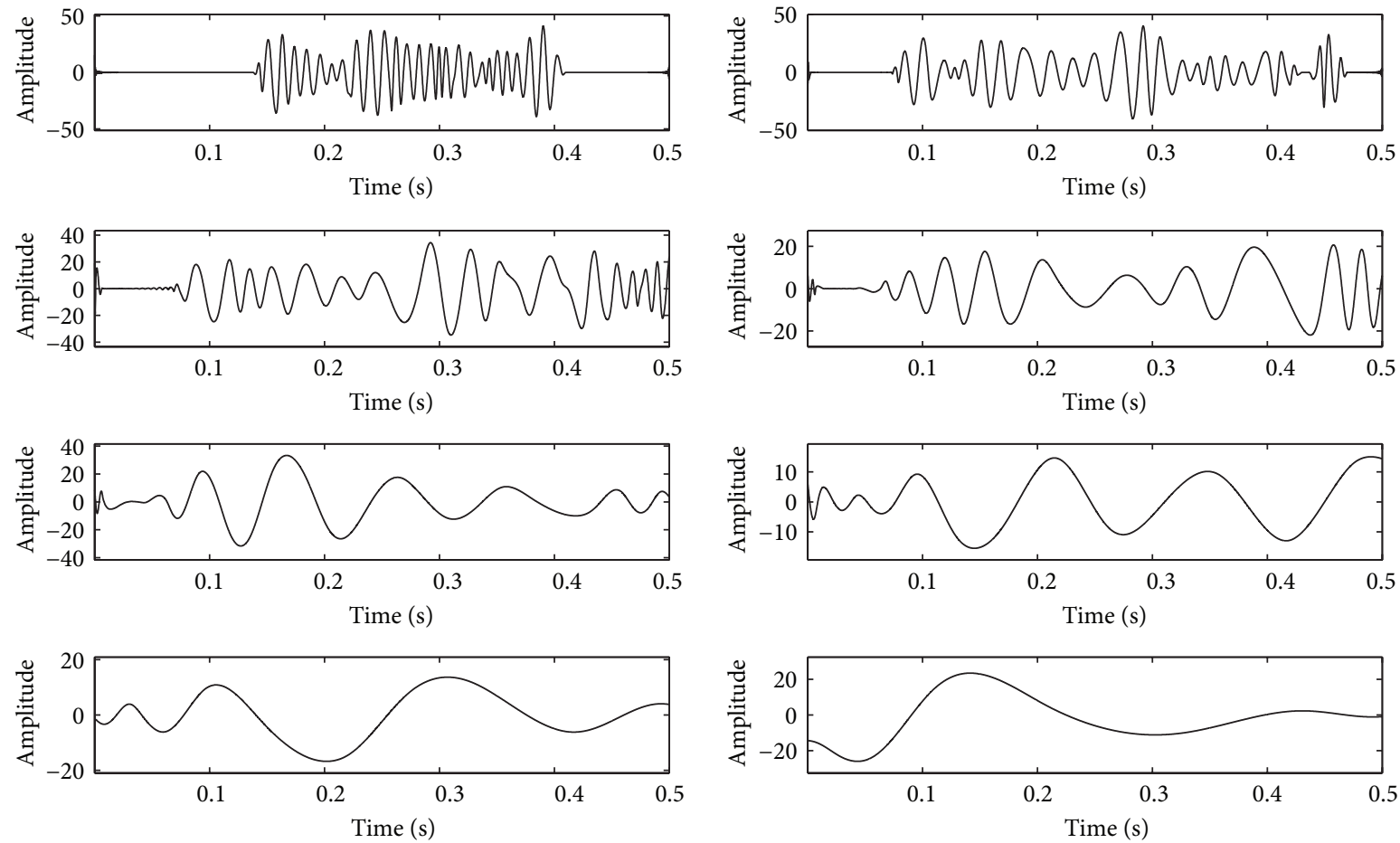

FIGURE 7: Time domain wave of IMFs based on EMD for the enveloped signal.
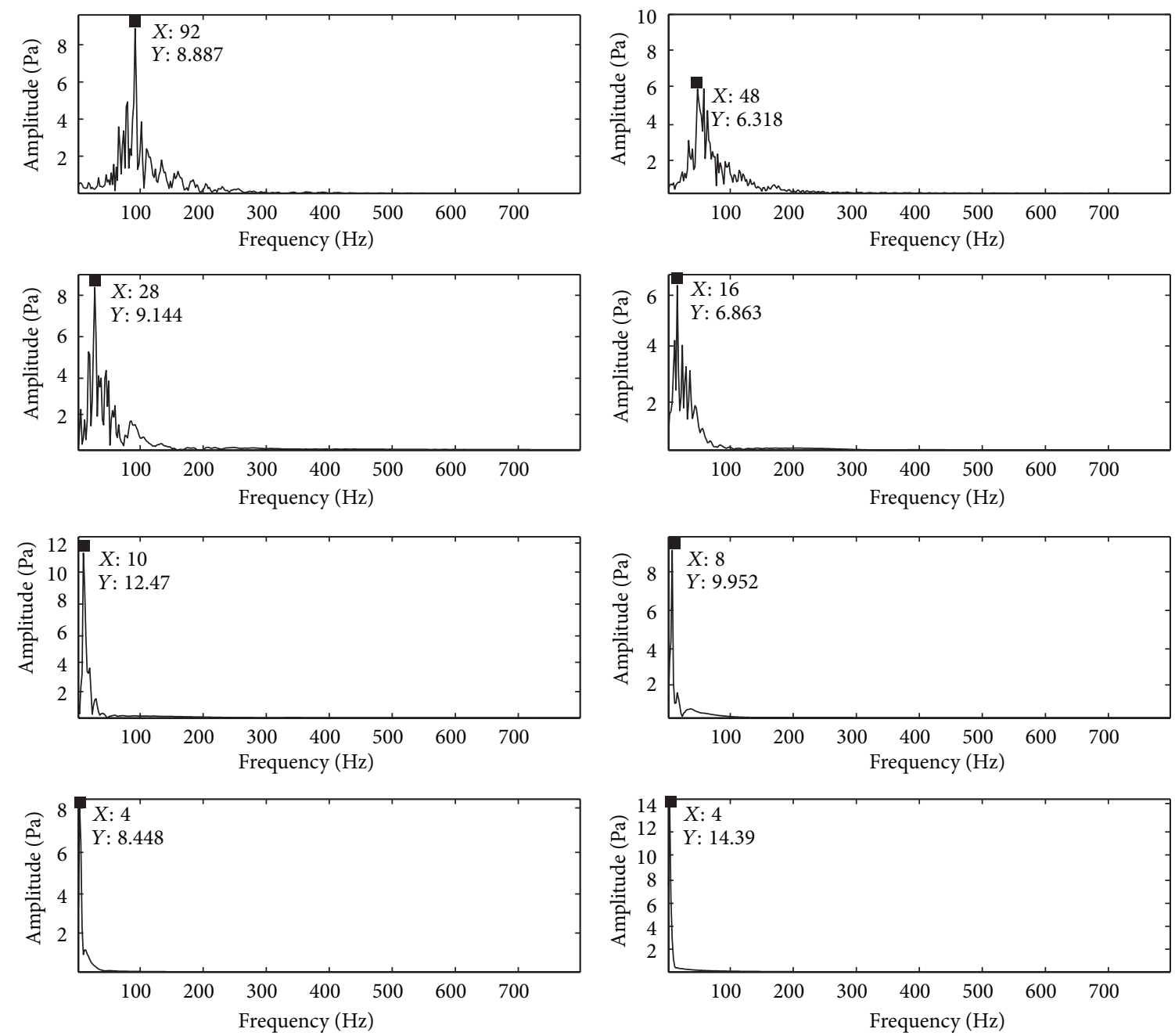

FIGURE 8: Spectrum of the IMFs for the envelope signal. 


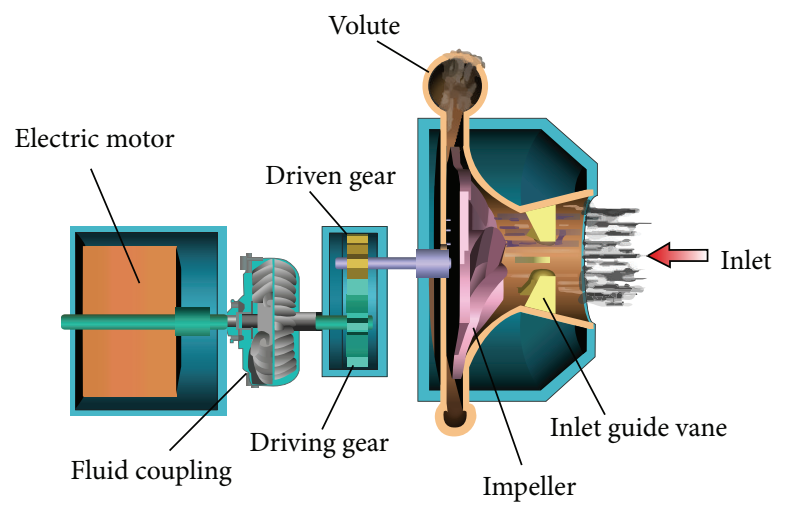

Figure 9: Experimental test-rig.
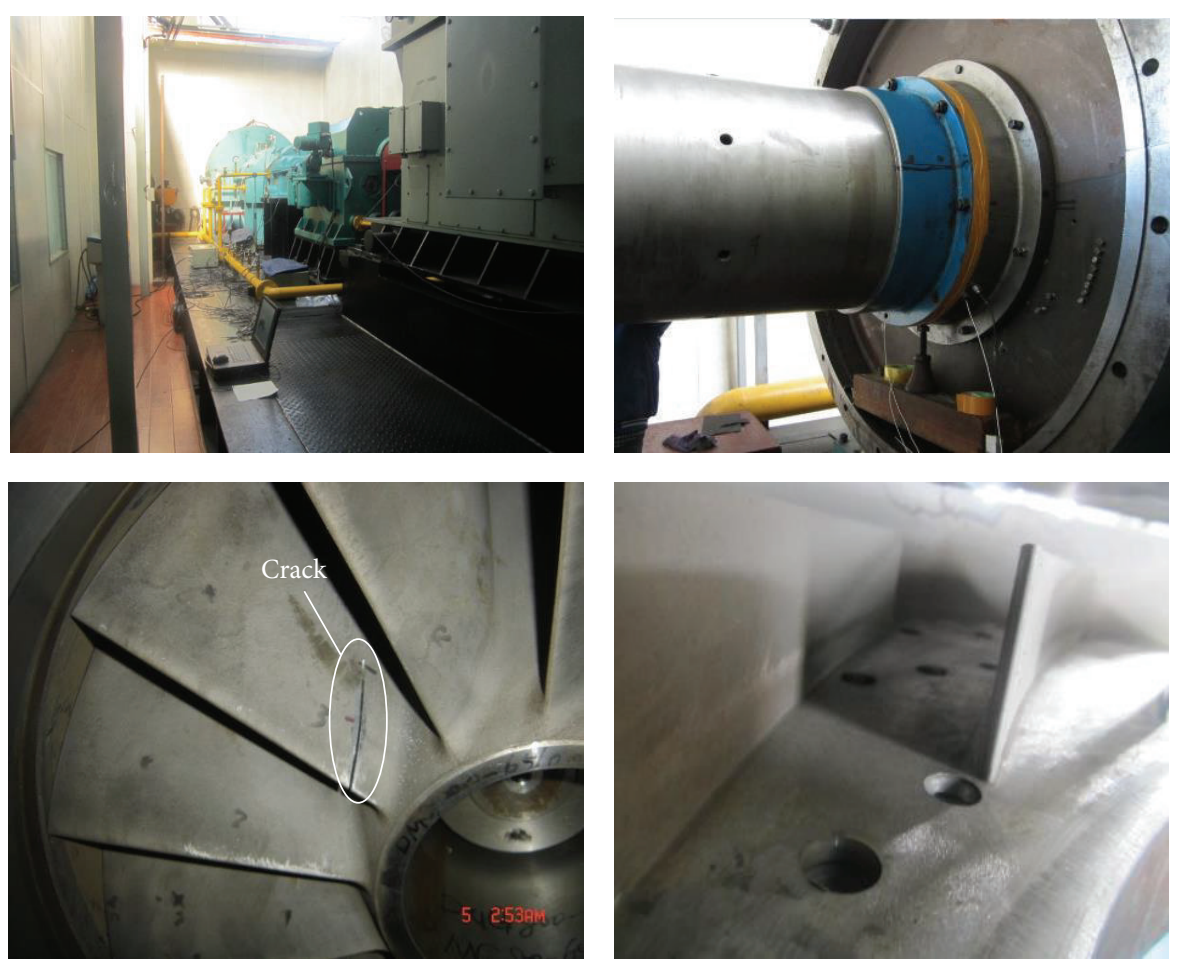

FIgURE 10: Pictures for test-rig.
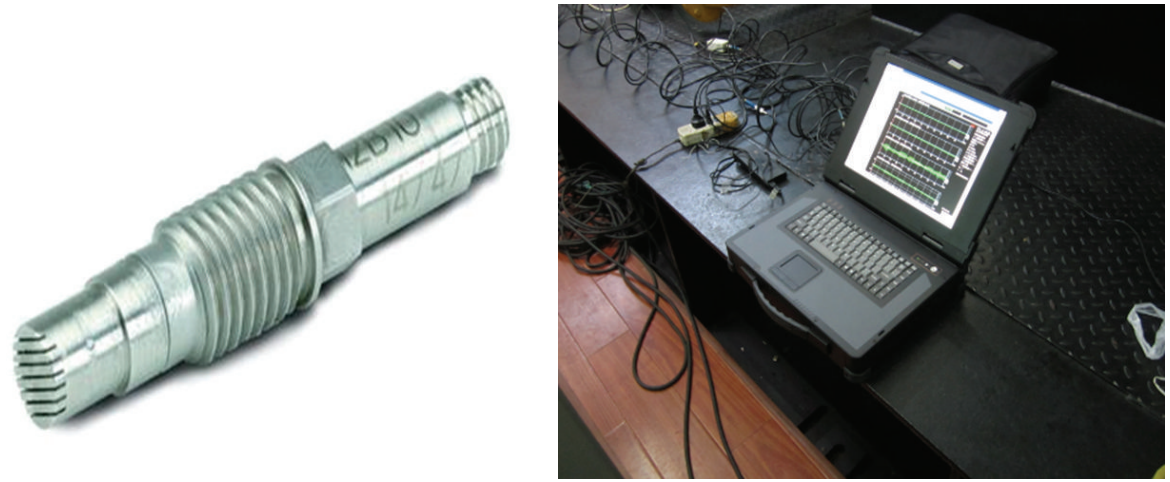

FIGURE 11: Pressure pulsation data acquisition system. 


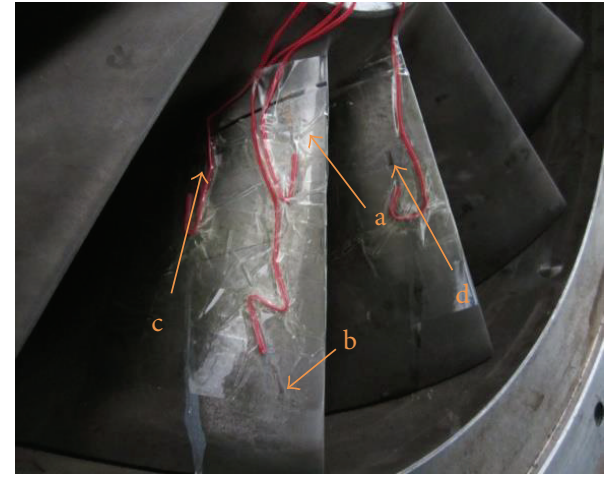

(a) Different strain sensor on the blade



(b) Position for data acquisition module

FIGURE 12: Strain testing process.

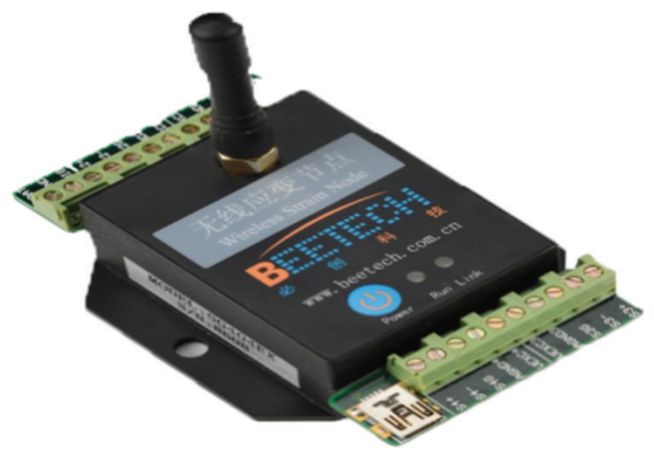

(a) Data acquisition module

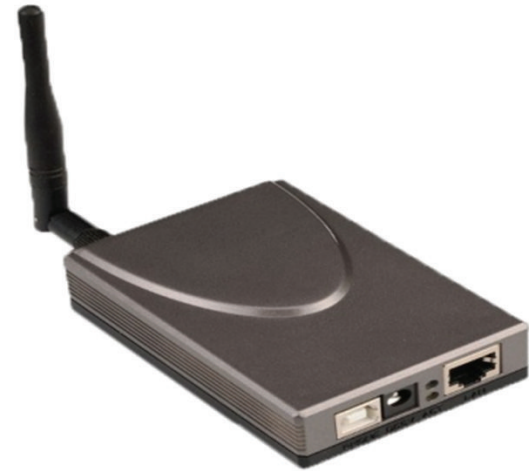

(b) Data receiving module

FIGURE 13: Strain data acquisition system.

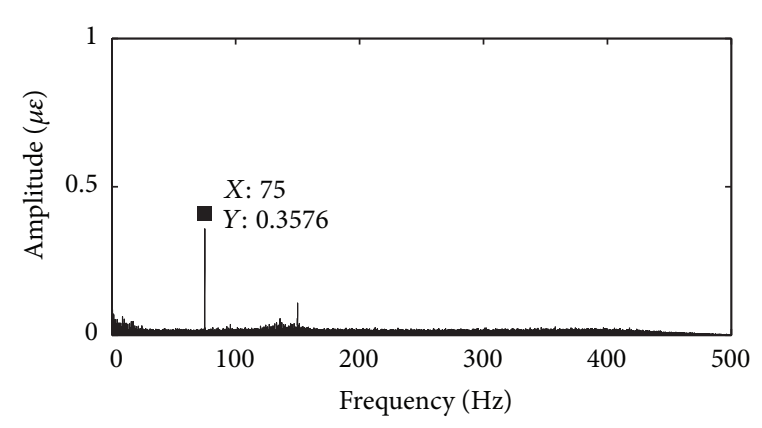

(a)

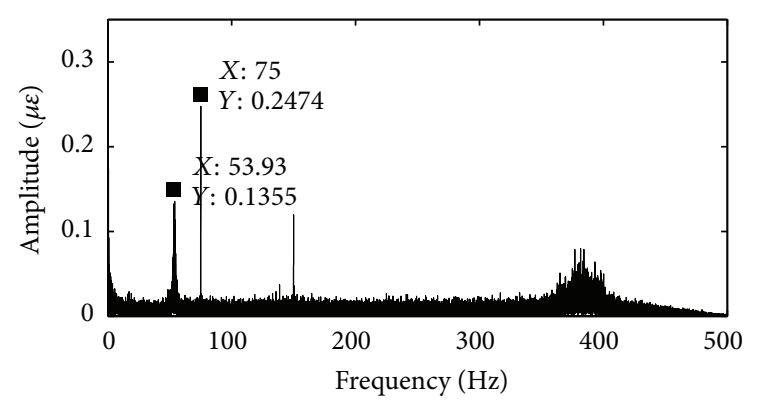

(c)

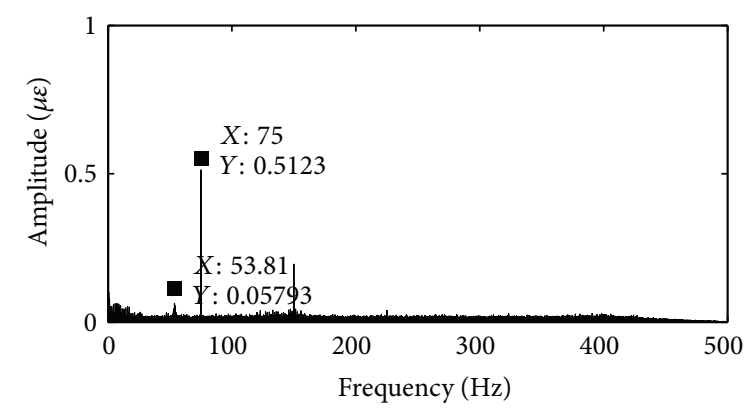

(b)

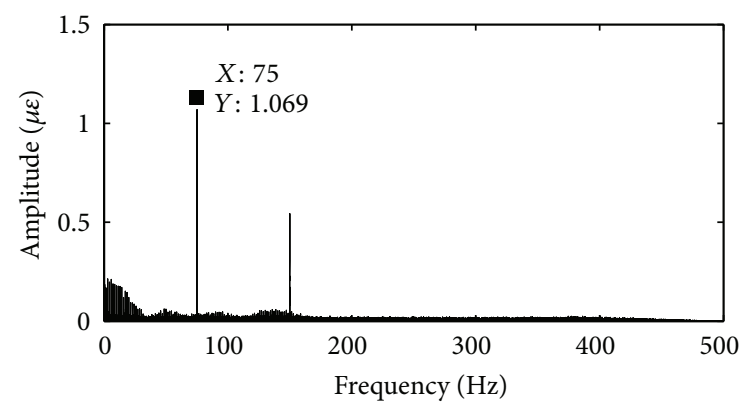

(d)

FIGURE 14: FFT Strain signal in 4500 RPM. 


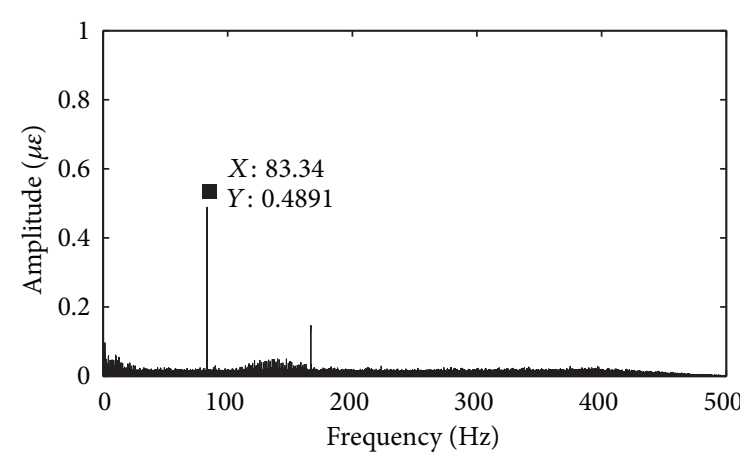

(a)

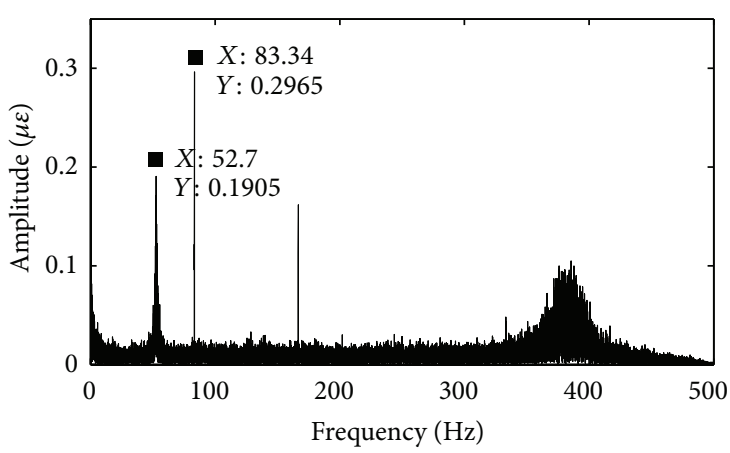

(c)



(b)

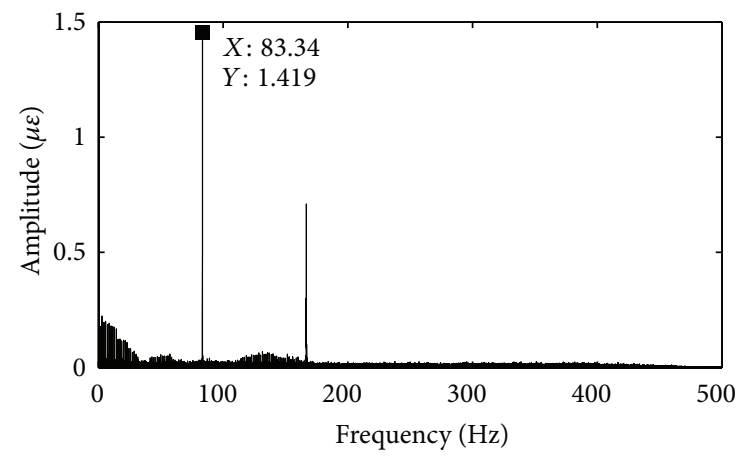

(d)

Figure 15: FFT Strain signal in 5000 RPM.

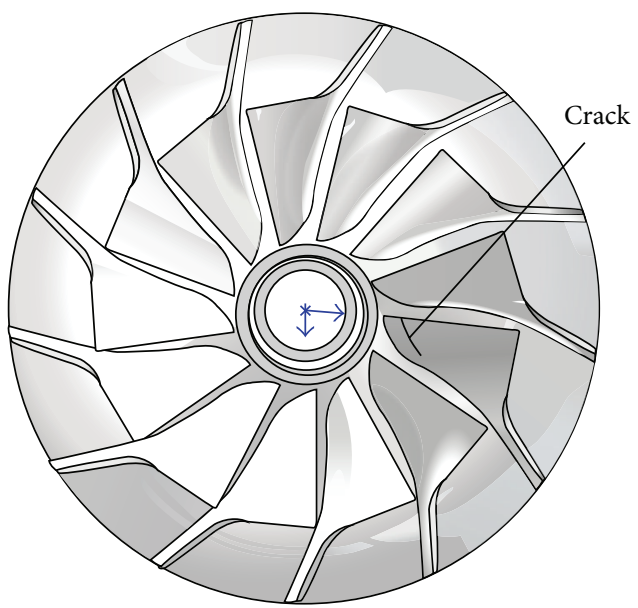

(a) Three models for impeller

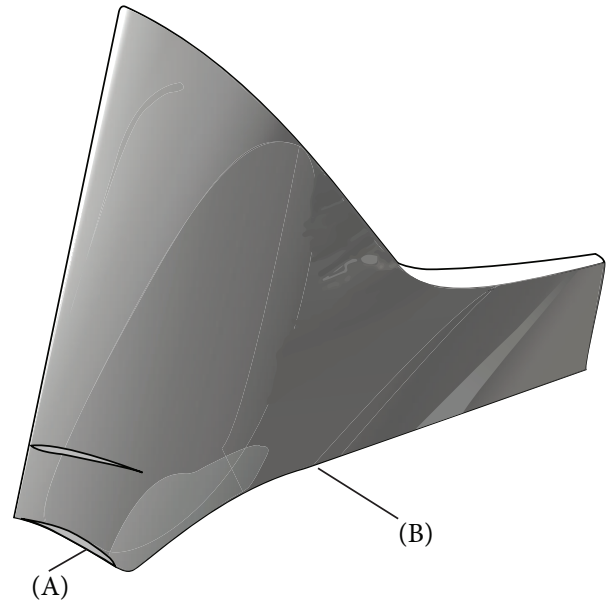

(b) A blade separated from impeller

FIgURE 16: Structure of impeller and blade.

TABLE 1: Characteristic parameters for $\varphi 800$ test-rig.

\begin{tabular}{lcc}
\hline Speed $(\mathrm{RPM})$ & 4500 & 5000 \\
Shaft frequency $(\mathrm{Hz})$ & 75 & 83.3 \\
Blade passing frequency $(\mathrm{Hz})$ & 975 & 1083 \\
\hline
\end{tabular}

4.2. Data Acquisition. There are three PP sensors produced by PCB Piezotronics (New York, USA) to monitor the working process; it is shown in Figure 11. One is installed in the inlet pipe; the other two are installed near the diffuser in the holes shown as Figure 10. The sensitivities of the PP sensors are $0.7044 \mathrm{mV} / \mathrm{Pa}, 0.9845 \mathrm{mV} / \mathrm{Pa}$, and $0.7336 \mathrm{mV} / \mathrm{Pa}$. PP signal, vibration signal is gathered by the NI-4472 data acquisition card. It is an 8-channel synchronous data gathering system. It is also shown in Figure 11.

The blade strain test is carried out by this research to verify the appearance of the fault frequency. The blade vibration will lead to the strain changes on the surface of 


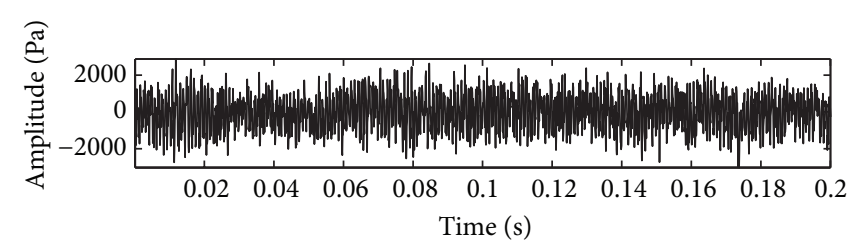

(a)

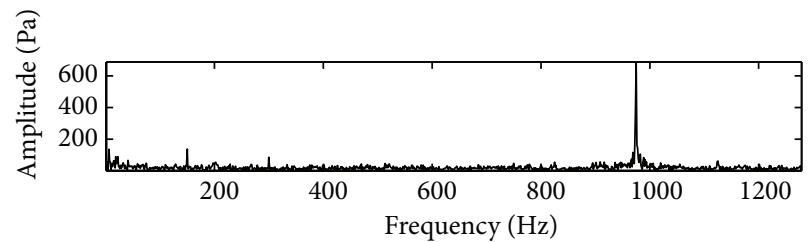

(b)

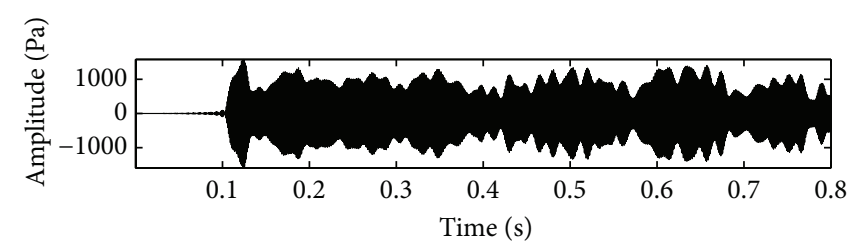

(c)

FIGURE 17: Time and frequency domain wave for the PP signal in 4500 RPM.
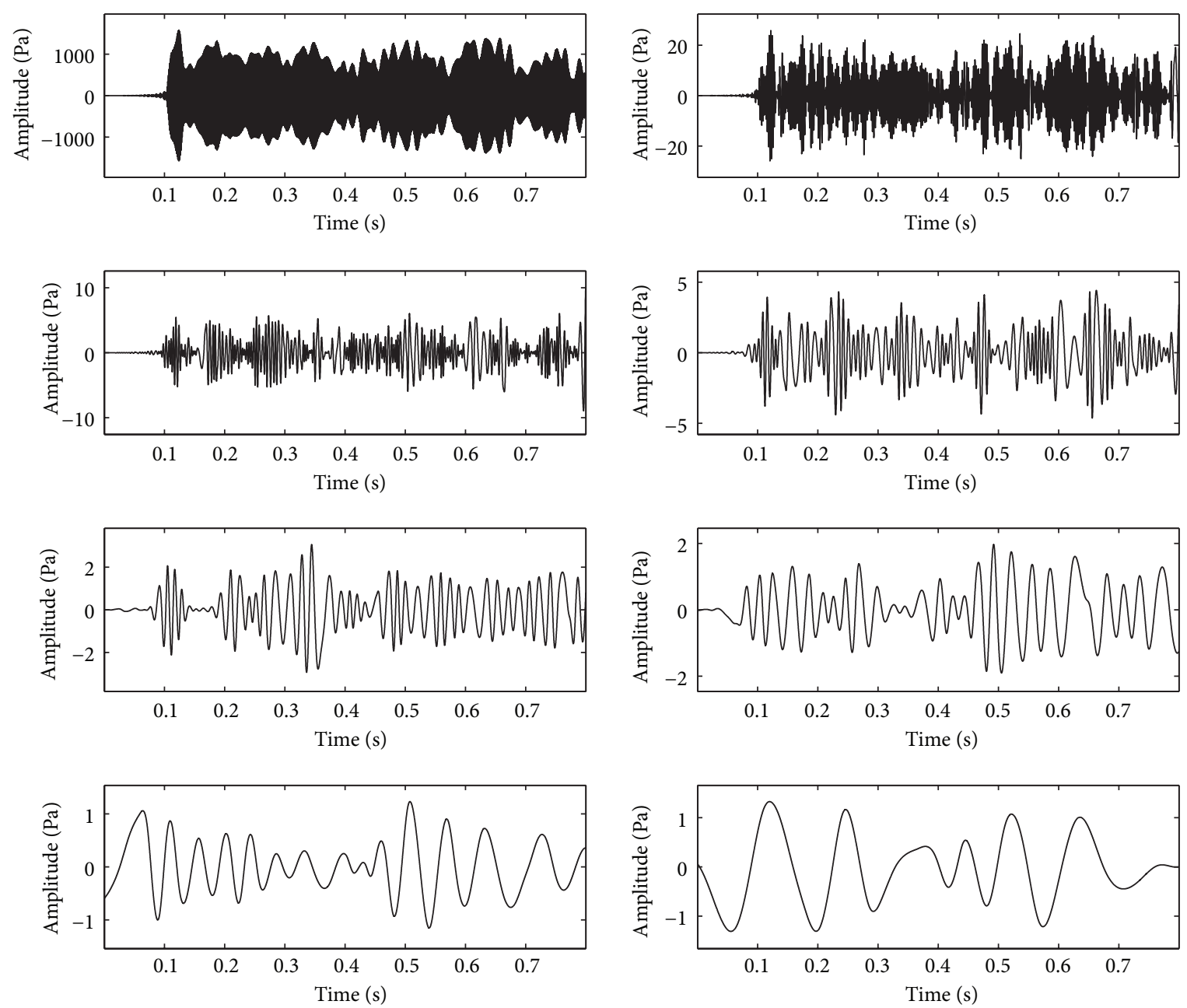

FIGURE 18: IMFs for the PP signal based on EMD in 4500 RPM. 

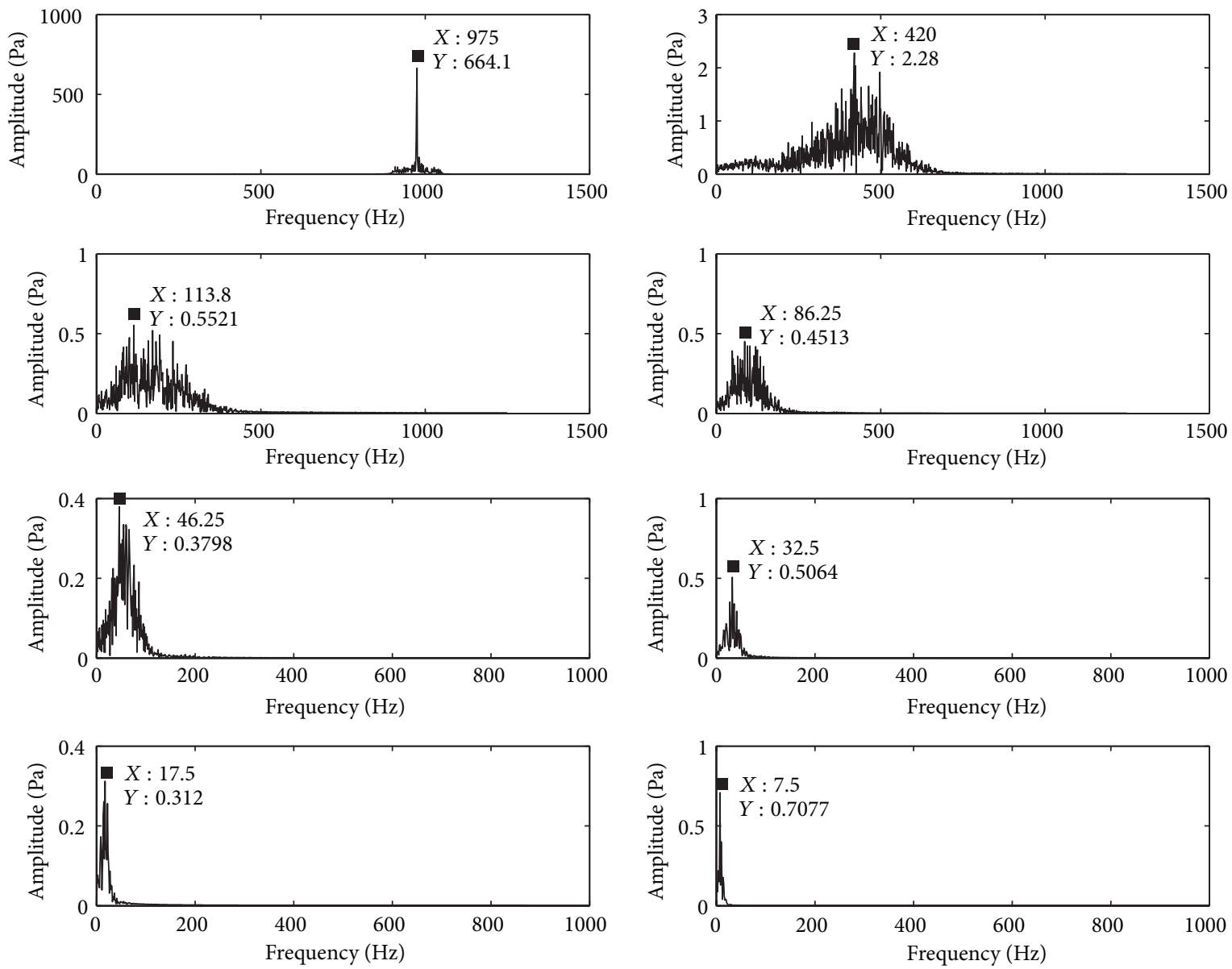

FIGURE 19: Spectrum for IMFs in 4500 RPM.
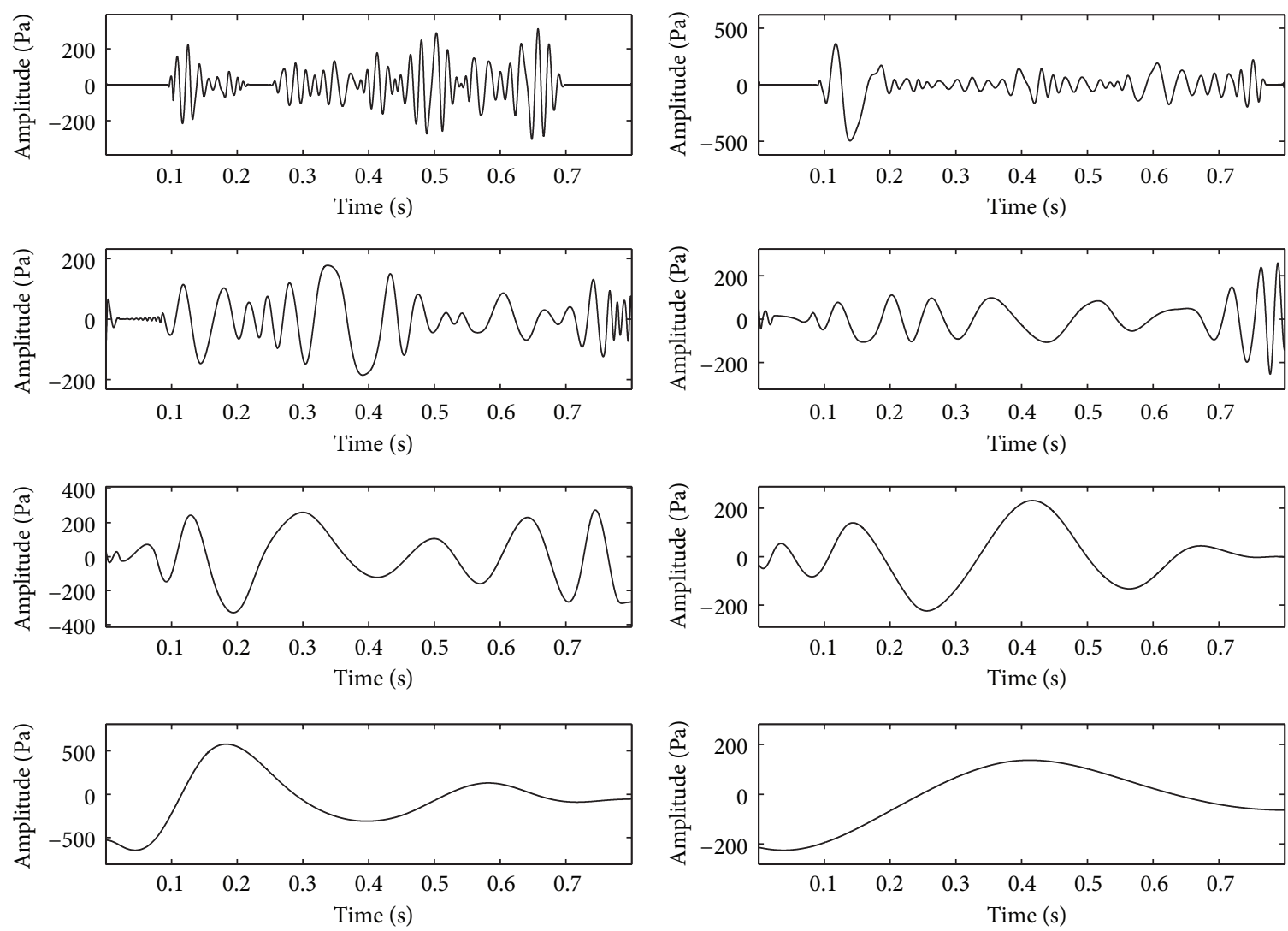

FIGURE 20: IMFs for the enveloped signal based on EMD in 4500 RPM. 

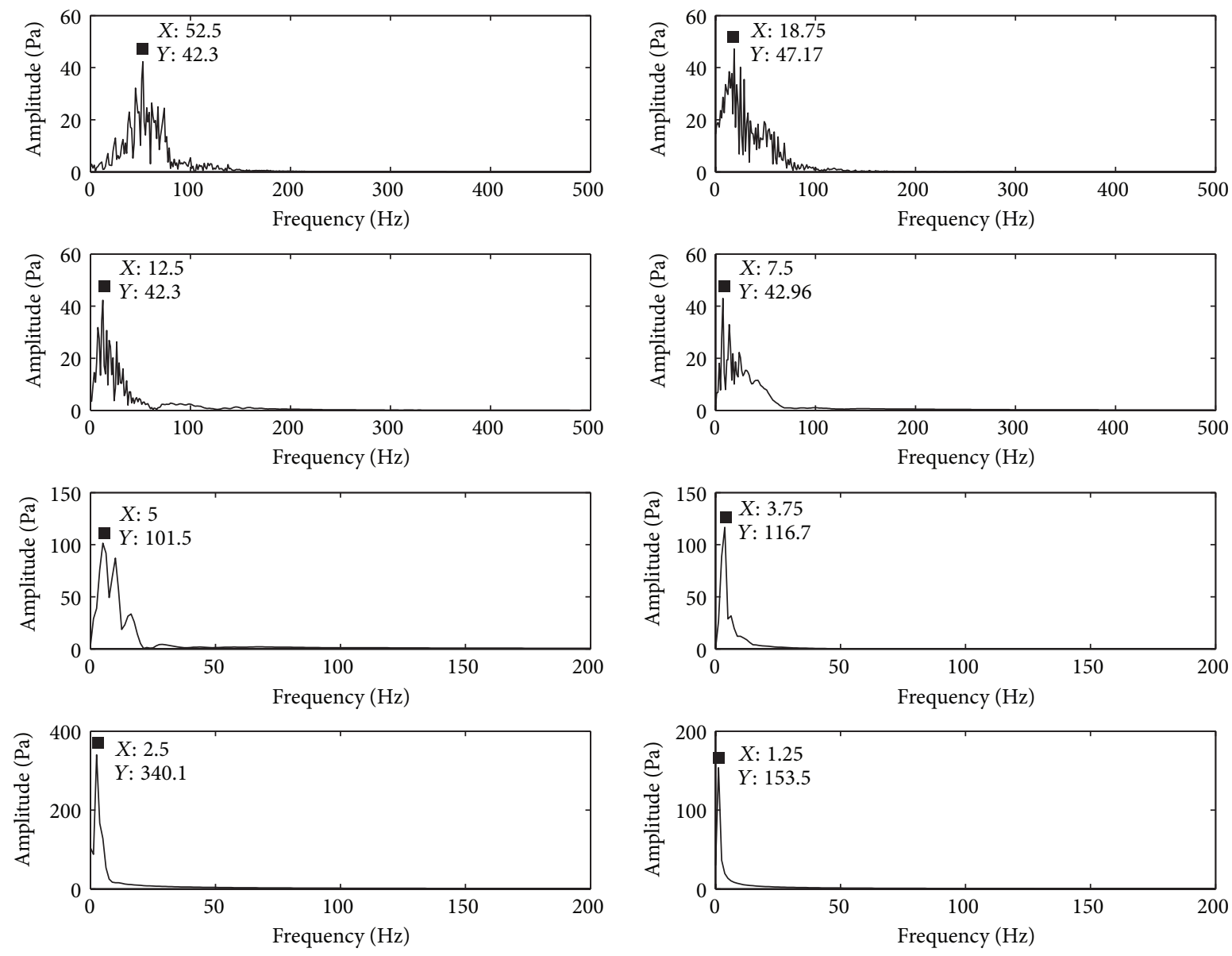

FIGURE 21: Spectrum for enveloped signal's IMFs in 4500 RPM.
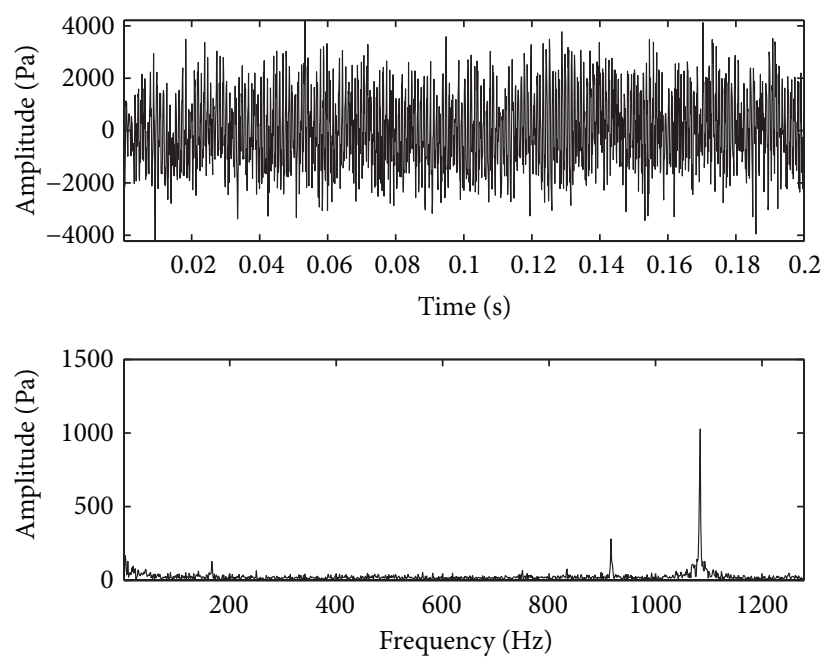

FIgURE 22: Time and frequency domain wave for the PP signal in 5000 RPM.

the blade, so the blade strain can reflect the blade vibration very well. When there are cracks on the blade, the blade vibration due to the change of the blade characteristics such as the stiffness and the blade stress vibration will change at the same time. So the stress test can be used to detect the blade crack. Due to real blade failure process, the location for the crack in selected near the hub shown in Figure 12. So the location for the crack is selected near the hub shown in Figure 12. To verify the appearance of the CF and its relationship with crack, the locations for strain gauge are shown in Figure 12(a). Points (a), (b), and (c) are on the crack blade. Point $(\mathrm{d})$ is on a normal blade. The data acquisition 

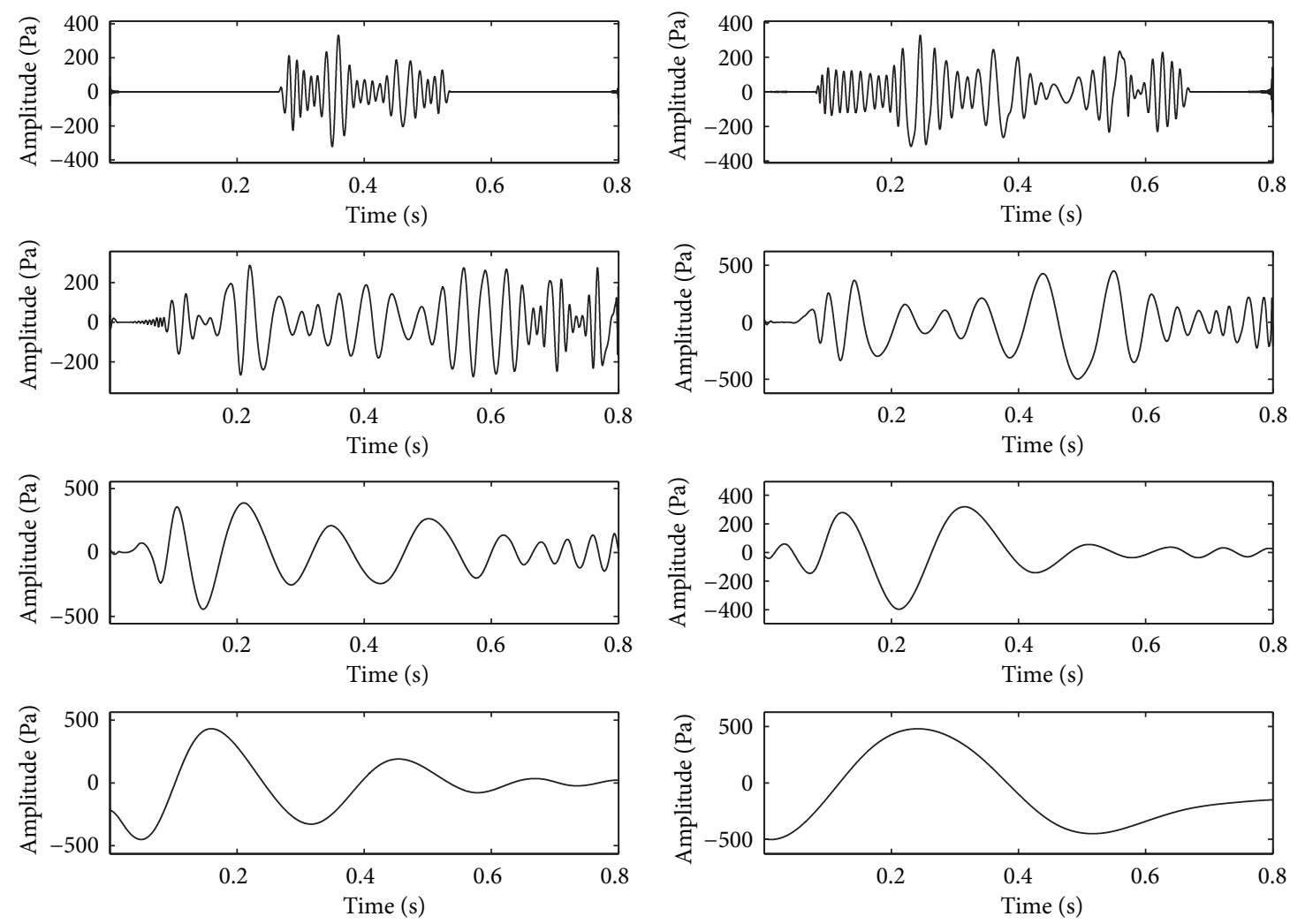

FIGURE 23: IMFs by EMD for the envelope signal in 5000 RPM.

module is shown in Figure 13(a) for launching data. It is also shown in Figure 12(b). Figure 13(b) presents the data receiving module. The data sampling frequency is $1024 \mathrm{~Hz}$ for strain signal. There are four channels to monitor the strain shown as Figure 12(a). It is important to determine the blade nonorder vibration as the reason of crack.

\section{Data Analysis}

5.1. Strain Signal Analysis. The frequency spectrum for the strain data with impeller speed $4500 \mathrm{RPM}$ is shown in Figure 14. The SF of the impeller is $75 \mathrm{~Hz}$. It is also clear because of the unbalance. The frequency $53 \mathrm{~Hz}$ is shown in the spectrum for point (b) and point (c). There is not the CF information for point (d) shown as Figure 14(d) because it is a normal blade. Point (c) is near the crack. It is clearer than point (b). It can be concluded that $53 \mathrm{~Hz}$ is the CF for blade vibration.

The same analysis is also carried on the impeller speed in 5000 RPM shown as Figure 15. Based on the above analysis process, the $\mathrm{CF}$ for blade nonorder vibration is $52.7 \mathrm{~Hz}$. The $\mathrm{CF}$ is almost the same as speed in $4500 \mathrm{RPM}$. Therefore, it can be concluded that $53 \mathrm{~Hz}$ is the CF for the crack. It is a nonorder vibration for the blade and the reason of crack. There is not CF for the normal blade. Strain analysis can help us to determine the CF for blade crack. As it is not convenient in real working condition for strain monitoring, feature extraction is important to obtain the CF from other monitored signals.

5.2. Pressure Pulsation Signal Analysis. PP signal is used to detect blade nonorder vibration information as for the crack. Compared the total length of the blade, the crack is very small (the diameter for the blade is $800 \mathrm{~mm}$ ) shown in Figure 16. Sides A and B in Figure 16(b) are together with impeller. They are not separated from impeller. It is just for clear demonstration with Figure 16(b). The impeller is manufacturing with whole milling process. At the same time, the averaging thickness of the blade is $10 \mathrm{~mm}$ to keep the stiffness of blade. Therefore, the information is weak for blade crack. It is the reason that it is difficult to determine the blade information. It can be just found when there is blade fracture. The crack information will be modulated to BPF as mentioned above although it is weak. There is not any modulated information in time domain shown in Figure 17(a). It is clear for BPF in the spectrum analysis shown in Figure 17(b). But it is not clear for the modulated frequency as the noise interference and nonorder vibration is very weak. It is impossible to obtain the modulated frequency. Therefore, the signal filter is investigated. The filter frequency band is $900-1050 \mathrm{~Hz}$. The filter signal is shown in Figure 17(c). The time domain and the frequency domain spectrum are shown in Figures 18 and 19, respectively. But there is not any information about the CF. 

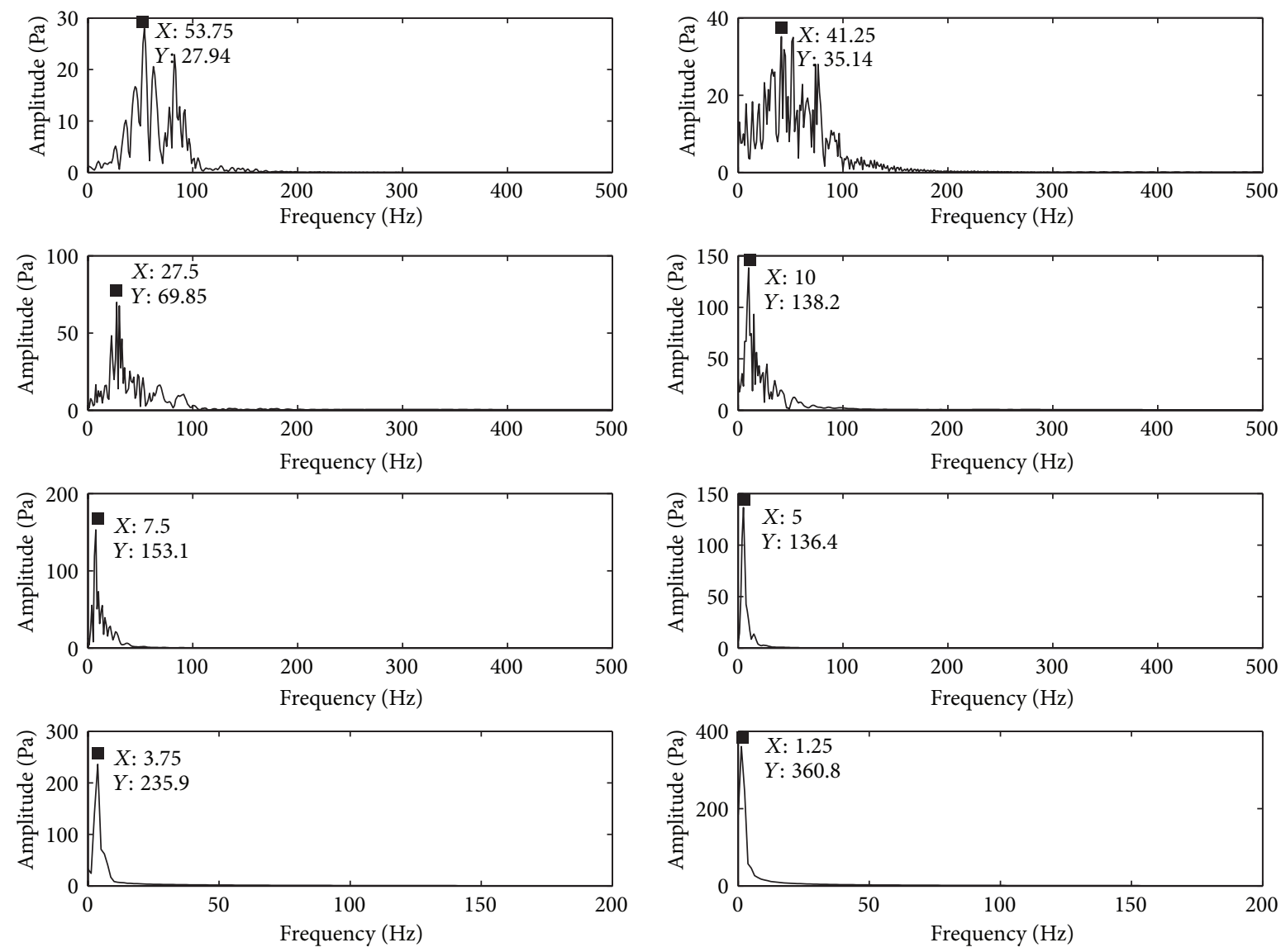

FIGURE 24: Time and frequency domain wave analysis for the different IMFs in 5000 RPM.

Envelope is used on the filtered signal. Then, EMD is used on envelope signal analysis. IMFs can be obtained shown in Figure 20. IMFs frequency spectrum analysis can clearly demonstrate the modulated frequency $53 \mathrm{~Hz}$ shown in Figure 21. Therefore, this method can be used to classify the crack CF for blade.

It is also with same result for $5000 \mathrm{RPM}$. Time and frequency analysis waveform for PP signal is shown in Figure 22. It is also difficult to recognize the CF. Therefore, signal filter is carried on. The filter frequency band is from $990 \mathrm{~Hz}$ to $1165 \mathrm{~Hz}$. Then, IMFs based on EMD for envelope signal are shown in Figure 23. IMFs spectrum analysis is shown in Figure 24. It is clear for the modulated frequency $53 \mathrm{~Hz}$. It has the same result with $4500 \mathrm{RPM}$. It can be verified that this method can effectively recognize the modulated nonorder vibration signal. It also demonstrates that this method can be used on feature frequency determination.

\section{Conclusions}

In this research, PP signals are used for blade crack condition monitoring and classification. The realization of this method is demonstrated in detail. Experiments on an industrial centrifugal compressor with a cracked blade were carried out to verify the effectiveness of this method. CF of blade crack information can be obtained by using EMD and spectrum analysis to obtain the modulated frequency. Strain signal is also investigated to monitor the crack CF. It is verified that crack characteristics can be determined by using PP signal. This research puts forward a method on how to determine the blade crack CF. Further investigations will also be carried on how to apply this method on real working condition blade crack classification. It will be helpful for blade crack early warning.

\section{Conflict of Interests}

The authors declare that there is no conflict of interests regarding the publication of this paper.

\section{Acknowledgments}

The work was supported by the Natural Science Foundation of China under Grant no. 51175057 and the Fundamental Research Funds for the Central Universities under Grant no. DUT14ZD204.

\section{References}

[1] Z. Y. Huang and X. F. Wang, Turbine Compressor, Chemical Industry Press, Beijing, China, 2004. 
[2] A. Kammerer, Experimental Research into Resonant Vibration of Centrifugal Compressor Blades, Swiss Federal Institute of Technology, Zürich, Switzerland, 2009.

[3] B. Wen, X. Wu, Q. Ding et al., Theory and Experiment of Nonlinear Dynamics for Rotating Machinery with Faults, Science Press, Beijing, China, 2004.

[4] M. Baumgartner, F. Kameier, and J. Hourmouziadis, NonEngine Order Blade Vibration in a High Pressure Compressor, ISABE, Melbourne, Australia, 1995.

[5] Y. G. Lei, J. Lin, Z. He, and D. Kong, "A method based on multisensor data fusion for fault detection of planetary gearboxes," Sensors, vol. 12, no. 2, pp. 2005-2017, 2012.

[6] N. J. Lourenço, M. L. A. Graça, L. A. L. Franco, and O. M. M. Silva, "Fatigue failure of a compressor blade," Engineering Failure Analysis, vol. 15, no. 8, pp. 1150-1154, 2008.

[7] A. Kermanpur, H. S. Amin, S. Ziaei-Rad, N. Nourbakhshnia, and M. Mosaddeghfar, "Failure analysis of Ti6Al4V gas turbine compressor blades," Engineering Failure Analysis, vol. 15, no. 8, pp. 1052-1064, 2008.

[8] F. L. Eisinger and R. E. Sullivan, "Vibration fatigue of centrifugal fan impeller due to Structural-Acoustic coupling and its prevention: a case study," Journal of Pressure Vessel Technology, vol. 129, no. 4, pp. 771-774, 2007.

[9] N. Roy and R. Ganguli, "Helicopter rotor blade frequency evolution with damage growth and signal processing," Journal of Sound and Vibration, vol. 283, no. 3-5, pp. 821-851, 2005.

[10] K. Elbhbah and J. K. Sinha, "Vibration-based condition monitoring of rotating machines using a machine composite spectrum," Journal of Sound and Vibration, vol. 332, no. 11, pp. 28312845, 2013.

[11] K. Saravanan and A. S. Sekhar, "Crack detection in a rotor by operational deflection shape and kurtosis using laser vibrometer measurements," Journal of Vibration and Control, vol. 19, no. 8, pp. 1227-1239, 2013.

[12] X. B. Liu, J. G. Lin, and Y. Wang, "Research on fault identification of blade crack of fan based on wavelet-packet analysis," Machine Tool \& Hydraulics, vol. 35, no. 9, pp. 241-243, 2007.

[13] A. Rama Rao and B. K. Dutta, "Vibration analysis for detecting failure of compressor blade," Engineering Failure Analysis, vol. 25, pp. 211-218, 2012.

[14] H. H. Yang, H. Hou, X. Y. Zeng, and J. C. Sun, "Fault diagnosis for fan based on auditory spectrum feature of sound signal," Chinese Journal of Scientific Instrument, vol. 30, no. 1, pp. 175179, 2009.

[15] L. Witek, "Experimental crack propagation and failure analysis of the first stage compressor blade subjected to vibration," Engineering Failure Analysis, vol. 16, no. 7, pp. 2163-2170, 2009.

[16] Y. Qu, C. Z. Chen, X. G. Zhao, and B. Zhou, "Wavelet scalogram identification for crack feature of wind turbine blade," Journal of Shenyang University of Technology, vol. 34, no. 1, pp. 22-47, 2012.

[17] X. Wang, H. Mao, H. Hu, and Z. Zhang, "Crack localization in hydraulic turbine blades based on kernel independent component analysis and wavelet neural network," International Journal of Computational Intelligence Systems, vol. 6, no. 6, pp. 1116-1124, 2013.

[18] B. C. Zhou, C. Zhang, and M. Yu, "Research on dynamic propagating characteristics of wind turbine blade's cracks," China Mechanical Engineering, vol. 24, no. 8, pp. 1108-1113, 2013.

[19] E. Egusquiza, C. Valero, X. Huang, E. Jou, A. Guardo, and C. Rodriguez, "Failure investigation of a large pump-turbine runner," Engineering Failure Analysis, vol. 23, pp. 27-34, 2012.
[20] N. E. Huang, Z. Shen, S. R. Long et al., "The empirical mode decomposition and the Hilbert spectrum for nonlinear and non-stationary time series analysis," The Royal Society of London. Proceedings. Series A. Mathematical, Physical and Engineering Sciences, vol. 454, no. 1971, pp. 903-995, 1998.

[21] A. Parey, M. El Badaoui, F. Guillet, and N. Tandon, "Dynamic modelling of spur gear pair and application of empirical mode decomposition-based statistical analysis for early detection of localized tooth defect," Journal of Sound and Vibration, vol. 294, no. 3, pp. 547-561, 2006.

[22] S. J. Loutridis, "Instantaneous energy density as a feature for gear fault detection," Mechanical Systems and Signal Processing, vol. 20, no. 5, pp. 1239-1253, 2006.

[23] B. Liu, S. Riemenschneider, and Y. Xu, "Gearbox fault diagnosis using empirical mode decomposition and Hilbert spectrum," Mechanical Systems and Signal Processing, vol. 20, no. 3, pp. 718734, 2006. 


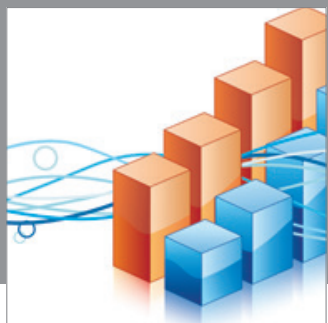

Advances in

Operations Research

mansans

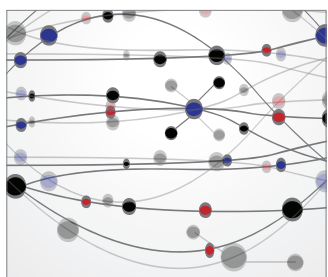

The Scientific World Journal
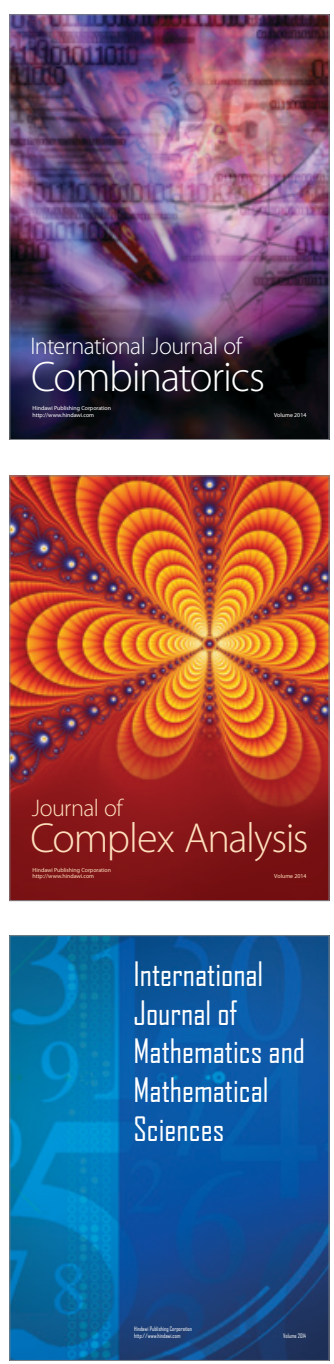
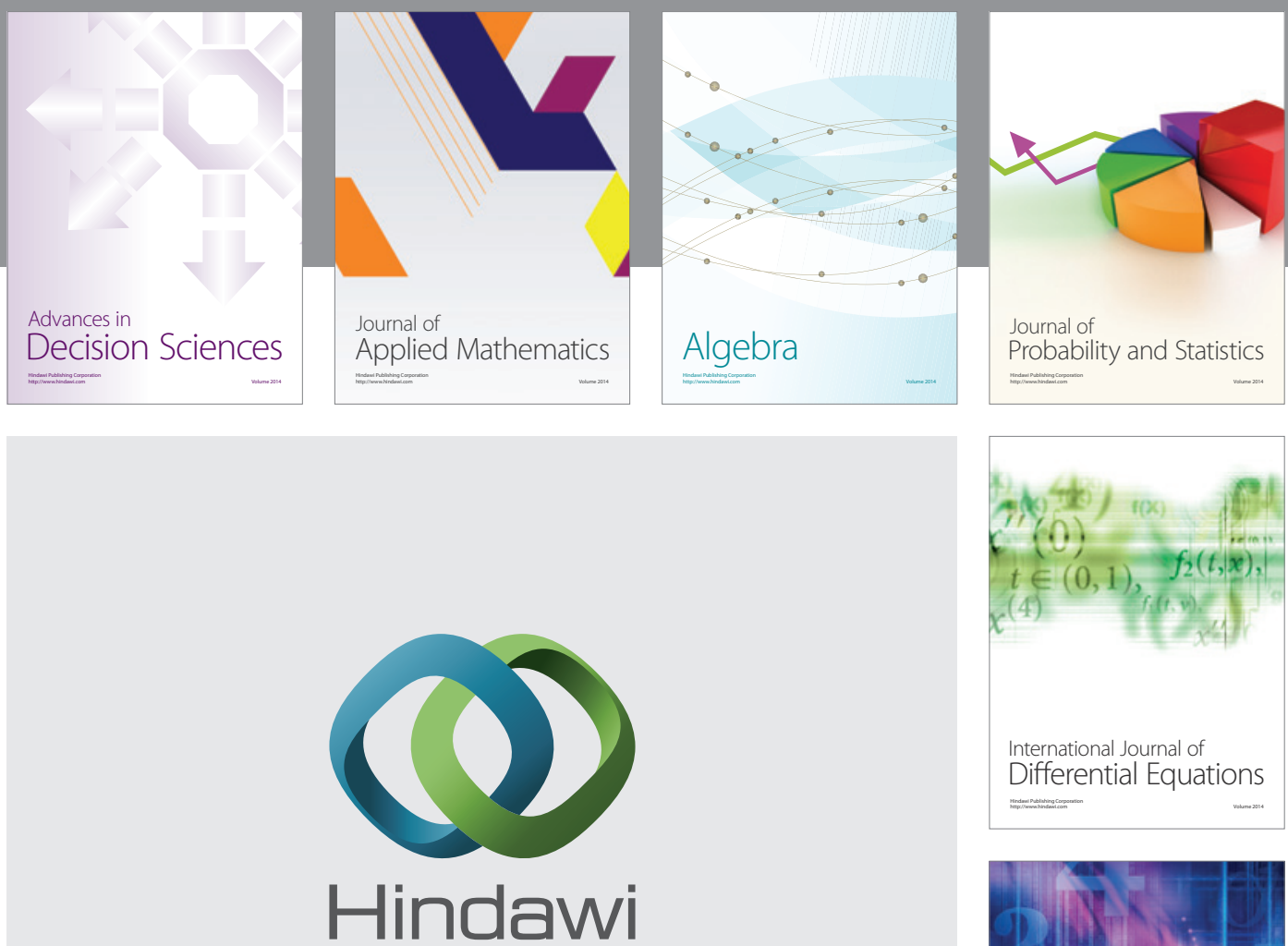

Submit your manuscripts at http://www.hindawi.com
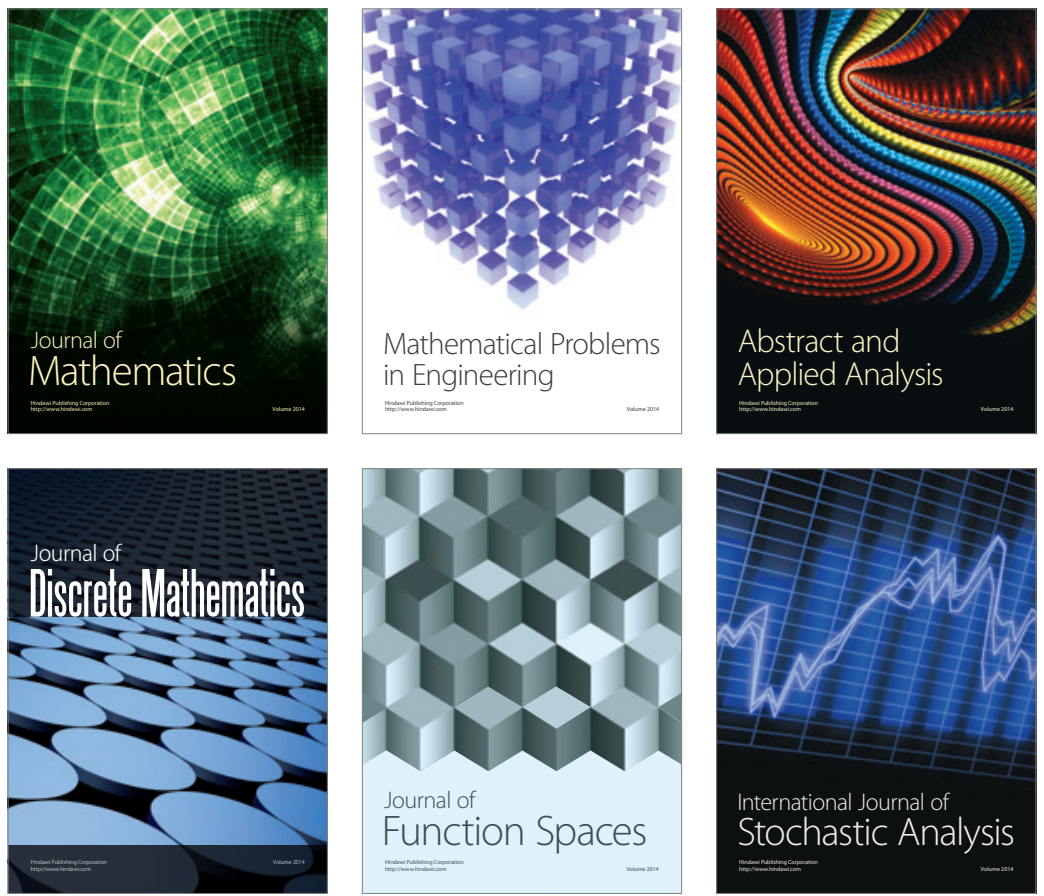

Journal of

Function Spaces

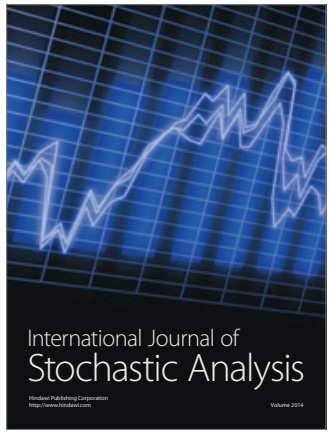

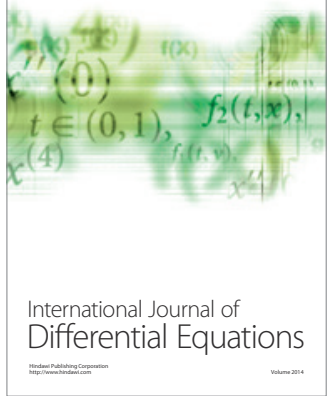
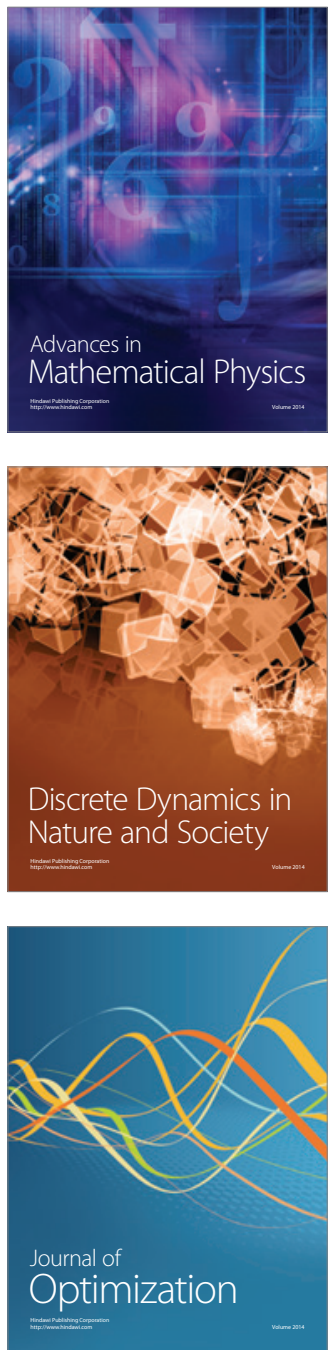